\section{Ärztebewertung im Netz: Gericht setzt Grenzen}

Grundsätzlich müssen Ärzte Kritik und Bewertungen im Internet hinnehmen. Aber: Wenn ein Patient dort falsche Angaben über eine Behandlung macht, können Ärzte dagegen vorgehen (Aktenzeichen 11 O 2608/12). Im Fall eines Zahnarztes haben Richter jetzt einem Portalbetreiber deutliche Auflagen gemacht. Ein Patient hatte sich über eine Implantatbehandlung beschwert. Der Zahnarzt konterte, in dem angegebenen Zeitraum habe er gar keine Implantatbehandlung durchgeführt und forderte die Löschung der Vorwürfe im Netz. Das Landgericht Nürnberg-Fürth gab ihm Recht. Martin Wortmann

\section{Medical Wellness und Prävention sind Zukunftstrends}

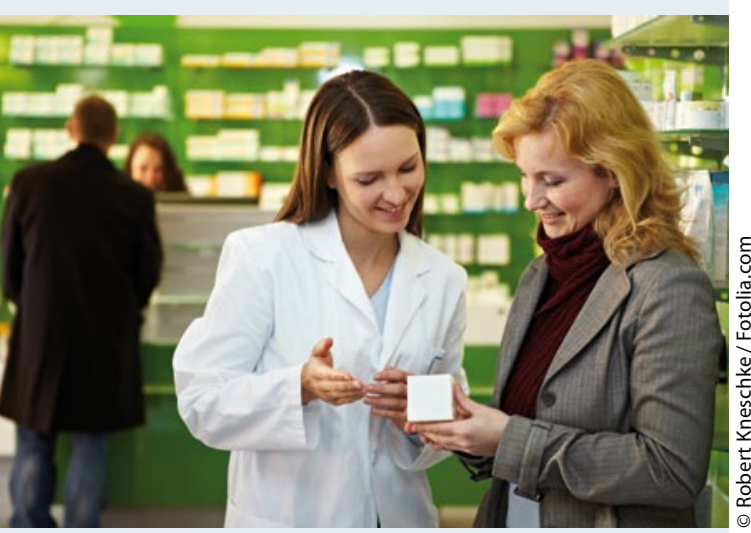

Die privaten Ausgaben für die Gesundheit werden weiter steigen.

Nach einer Studie der Berenberg Bank und des Hamburgischen Weltwirtschaftsinstituts (HWWI) steigt der Trend zu Medical Wellness und Prävention. Privat finanzierte Gesundheitsausgaben werden in Zukunft weiter wachsen. Die Weltbevölkerung wächst, die westlichen Gesellschaften altern, die Einkommen steigen und die Gesundheitsausgaben nehmen überproportional zum Einkommen zu, wie Wirtschaftsforscher prognostizieren. Mit ihren privaten Ausgaben sorgen schon jetzt viele Menschen dafür, dass die Wachstumsraten im Gesundheitsmarkt deutlich über jenem des durchschnittlichen Wirtschaftswachstums liegen.

Dirk Schnack

\section{Im Zweifelsfall ist rechtlicher Beistand immer empfehlenswert}

\author{
Wenn ein Arzt mit seiner KV um viel Geld streitet, gibt es kaum einen Grund \\ auf den Rechtsanwalt zu verzichten.
}

treitet ein Arzt mit seiner KV um viel $\checkmark$ Geld, dann darf er sich in der Regel auch rechtlichen Beistand holen. Soweit die KV nachgibt, muss sie dann auch im Widerspruchsverfahren den Anwalt bezahlen. So urteilte kürzlich der Vertragsarztsenat des Bundessozialgerichts (BSG) in Kassel (Aktenzeichen B 6 KA 43/11 R).

\section{Rechtsbeistand bei großer wirtschaftlicher Tragweite}

Im konkreten Fall hatte die KV BadenWürttemberg die Abrechnungen eines Radiologen für 2003 und 2004 sachlichrechnerisch richtiggestellt und danach fast $155.000 €$ zurückverlangt. Für das Widerspruchsverfahren ließ sich der Arzt von einem Anwalt beraten. Der Widerspruch war in vollem Umfang erfolgreich. Allerdings weigerte sich die KV, auch den Rechtsanwalt zu bezahlen. Das müsse sie aber, mahnte das BSG. Ob das Hinzuziehen eines Anwalts notwendig war und daher von der unterlegenen KV zu bezahlen ist, sei immer aus der Sicht des Arztes zu entscheiden. Das sei in der Regel zu bejahen, wenn, wie in diesem Fall, der Streit „von nicht unerheblicher wirtschaftlicher Tragweite ist". Gleiches gelte, wenn es um „Auslegungsfragen zu den Leistungslegenden der Gebührenordnungen“" geht.

Verzichtbar sei ein Rechtsanwalt lediglich dann, wenn der Arzt in der Vergangenheit bereits Erfahrungen in der strittigen Frage gemacht hat oder wenn es um einen offensichtlichen Fehler der KV geht, auf den der Arzt lediglich hinweisen muss. Dagegen spiele es keine Rolle, ob der Anwalt im Widerspruchsverfahren eine eigene Stellungnahme abgegeben hat, betonten die Kasseler Richter.

\section{Abrechungstipp}

\section{Wann die GKV die Akupunktur bezahlt}

Nach den Vorgaben des EBM sind Akupunkturleistungen, soweit ein Arzt die Genehmigung der KV besitzt, lediglich bei chronischen Schmerzen infolge einer Gonarthrose eines oder beider Kniegelenke und bei chronischen Schmerzen der LWS zu Lasten der GKV abrechenbar. In diesen Fällen ist eine Akupunkturbehandlung nach Nr. 30791 bis zu zehnmal, mit Begründung bis zu 15mal im Krankheitsfall abrechenbar, und zwar getrennt für jede der beiden Indikationen. Im Gegensatz dazu kann man die Abrechnung der Nr. 30790 unabhängig von der Diagnose nur einmalig pro Krankheitsfall in Rechnung stellen - auch wenn beide Indikationsgebiete getrennt behandelt werden. Zur Rekapitulation: Der Krankheitsfall beinhaltet das laufende und die drei nachfolgenden Kalendervierteljahre (EBM, Allg. Best. 3.2).

Das heißt aber auch, dass bei Behandlung eines Kniegelenks eine später notwendige Behandlung des anderen Kniegelenks erst zwölf Monate nach der ersten Akupunkturserie abgerechnet werden kann. Eine eventuell erforderliche und indizierte Akupunkturbehandlung der LWS kann jedoch vor Ablauf des Krankheitsfalles mit der Nr. 30791 zu Lasten der GKV durchgeführt werden. Ein Punkt des obligaten Leistungsinhalts der Nr. 30790, der nicht selten vergessen wird, ist der Bericht an den Hausarzt, dem der Patient allerdings schriftlich zustimmen muss (EBM Allg. Bestimmungen 2.1.4).

Festzuhalten bleibt auf jeden Fall, dass eine Akupunkturbehandlung nur in eng begrenztem Ausmaß auf Kosten der GKV abrechenbar ist. Alle anderen oder zusätzlichen Akupunkturbehandlungen sind IGeL und nach GOÄ abrechenbar, ohne dass irgendwelche Genehmigungen eingeholt werden müssen.

Dr. med. Heiner Pasch 